\title{
Computational Modelling of the Role of Atrial Fibrillation on Cerebral Blood Perfusion.
}

\author{
Timothy J Hunter ${ }^{1,2}$, Jermiah J Joseph ${ }^{1,2}$, Udunna Anazodo ${ }^{1,2}$, Sanjay R Kharche ${ }^{1,2}$, \\ Christopher W McIntyre ${ }^{1,2}$, and Daniel Goldman ${ }^{1}$ \\ ${ }^{1}$ Department of Medical Biophysics, University of Western Ontario, London ON N6A 3K7, \\ Canada. \\ ${ }^{2}$ Lawson Health Research Centre, London ON N6A 5W9, Canada. \\ dgoldma2@uwo.com
}

\begin{abstract}
Atrial fibrillation is a prevalent cardiac arrhythmia, and may reduce cerebral blood perfusion augmenting the risk of dementia. It is thought that cerebral arterial geometry variants play an important role in cerebral perfusion. This computational work investigated the role of geometric variants on cerebral blood flow in the presence of cardiac atrial fibrillation.

A model consisting of a detailed cerebral and whole-body circulation, along with baroreflex control mechanism was developed. Cerebral perfusion based on vasculature geometry variations, represented by Circle of Willis variants, was simulated in the presence of atrial fibrillation conditions. Perfusion and its heterogeneity were quantified using segment-wise hypoperfusion events and mean perfusion at terminals.

It was found that cerebral perfusion and the rate of hypoperfusion events strongly depends geometry variation as well as atrial fibrillation induced stochastic heart rates. The hypoperfusion events were specific to particular arteries in each variant. Our results, based on biophysical principles, suggest that cerebral vascular geometries modulate the impact of atrial fibrillation in cerebral perfusion. Further, our findings suggest potential clinical assessment sites.
\end{abstract}

Keywords: 0D model, atrial fibrillation, cerebral blood flow.

\section{Introduction}

Atrial fibrillation (AF) is known to reduce cerebral perfusion [1]. Ongoing imaging research strongly suggests that a disrupted cerebral blood flow promotes debilitating dementia [2]. The effects of AF on cerebral perfusion may be modulated by cerebral vascular geometry, and specifically by cerebral Circle of Willis (CoW) variants [3].

Multi-scale hemodynamic modelling has been used to study cerebral circulation to gain insights into patient specific hemodynamics [4]. While 3D modelling provides accuracy and realistic patient specific assessment, current methods remain computationally resource intensive. In contrast, lumped parameter (0D) models are known to also provide clinically relevant information for a fraction of the resources and time [5]. We 
have previously used lumped parameter models to gain insights into the causes of pediatric hypertension [6] and investigate therapeutic hypothermia. 0D models have also been used to understand the interplay between AF and cerebral vasculature [7]. However, the role of cerebral vascular structural variants, i.e. CoW variants, in AF-cerebral perfusion relationship remains underexplored. In this study, a composite lumped parameter (0D) model of human circulation with detailed cerebral vasculature was developed to permit uncovering of the relationship between AF, CoW variants, and cerebral perfusion.

\section{Methods.}

An established OD lumped parameter whole body circulation model [8,9] was coupled to a baroreflex control model [10] in a recent study, which was further developed to incorporate detailed cerebral vasculature [5] descriptions (Fig. 1).
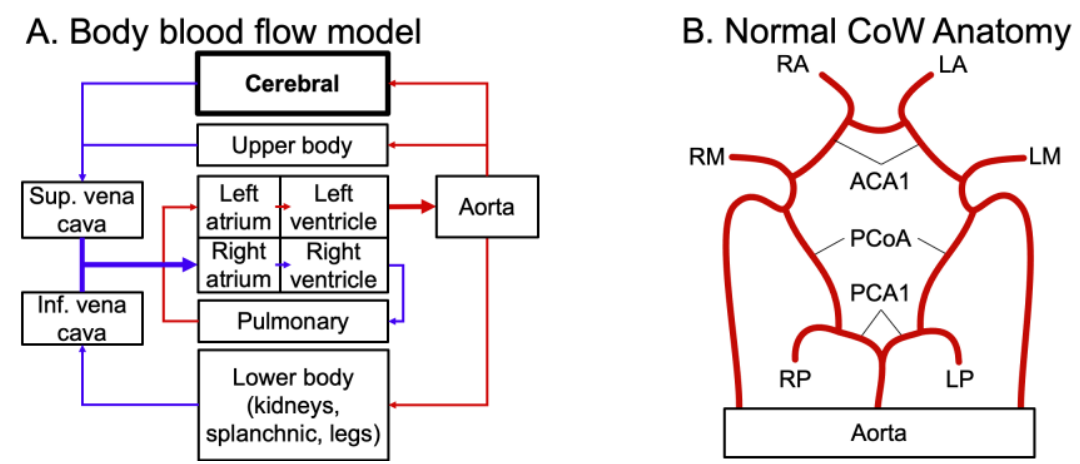

Fig. 1. Components of the hemodynamic model. A: Caricature of the whole-body blood flow model. The cerebral model (black box, top) is expanded in the right (B) panel. B: A representative cerebral arterial architecture consisting of Circle of Willis. RA: Right anterior artery; LA: left anterior artery; RM: right middle artery; LM: left middle artery; RP: right posterior artery; LP: left posterior artery; ACA1: pre-communicating anterior cerebral artery; PCoA: posterior communicating artery; and PCA1: pre-communicating posterior cerebral artery.

The model simulates blood flow using the Windkessel approach, with resistances (blood viscosity, length, and diameters) and capacitances (blood vessel elasticity). The heart was modelled as a four chamber system, with each providing a pumping function based on respective time-varying elastances [9]. Blood flow from the aorta was permitted to flow into the brain by means of three inlets represented by RP in Fig. 1, B. The cerebral outlets were drained into the low pressure superior vena cava (Fig. 1, A). The baroreflex control model was modelled as a system of first-order low-pass filters that used aortic pressure to modulate all systemic resistances, capacitances, and maximum ventricular elastances.

This work considers the 6 most common variants of the Circle of Willis found in the cerebral vasculature. The first variant, termed normal is represented in Fig. 1, B. The second variant has ACA1 artery missing, third has PCA1 missing, fourth has one PCoA missing, fifth is both left and right PCoA missing, and sixth is PCoA and contralateral 
PCA1 (PCoA + P1) missing [11]. CoW variants were modelled by blocking flow in relevant arterial segments in the normal model (Fig. 1, B).

AF was modelled by assigning stochastic RR intervals centered around a mean heart rate, modifying ventricular elastances (contractility) [12], and assigning nil atrial contractility $[7,13,14]$. Each simulation was performed at five different intrinsic heart rates $(50$ to $90 \mathrm{bpm})$ in accordance with clinical practice [15]. The Gaussian distributions underlying the RR intervals and the representative RR interval time series are illustrated in Fig. 2.
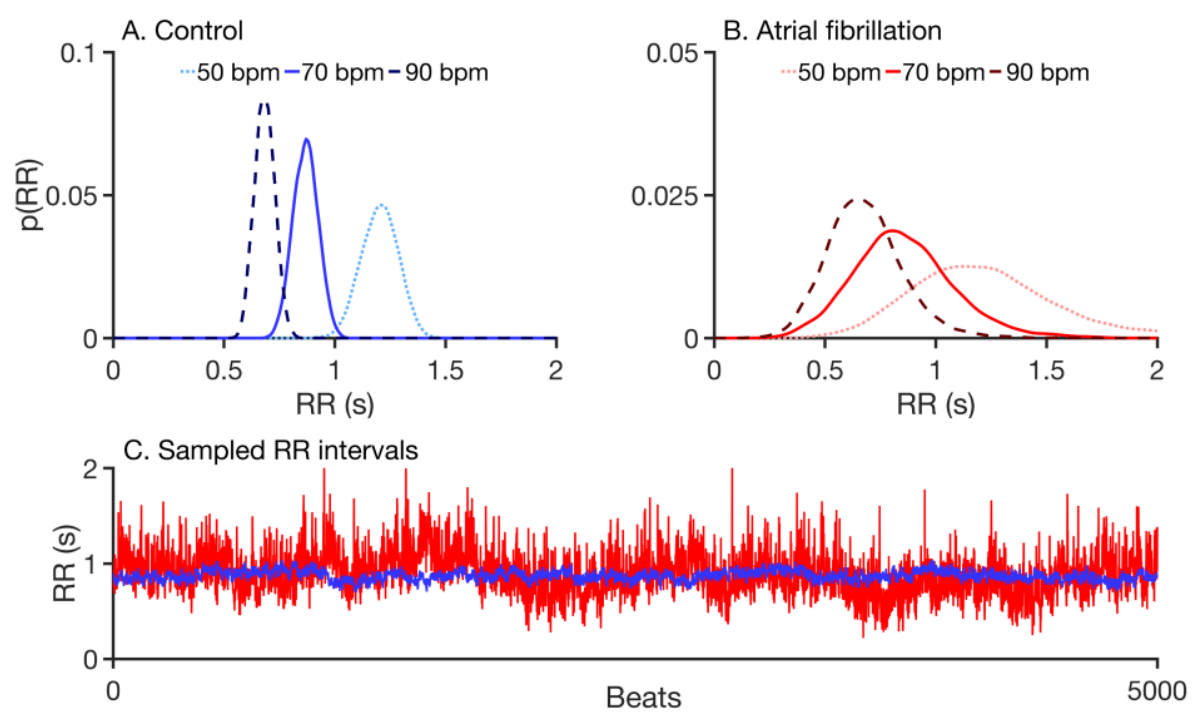

Fig. 2. Stochastic RR interval assignment. Top row: Probability distribution functions for sampled RR intervals in NSR (A), and AF (B) at shown heart rates. C: Sampled RR intervals at 70 bpm over the span of 5000 beats under NSR (blue line) and AF (red line) conditions.

Each simulation was performed under normal (NSR) and AF conditions. In each simulation, the number of hypoperfusion events were recorded to represent cerebral perfusion deficit. Derived measurements were the number of hypoperfusion events in each vascular bed over the 5000 beats of the simulation. A hypoperfusion event in any vascular bed was defined as a heartbeat in which the mean blood flow through the vascular bed falls below the $5^{\text {th }}$ percentile of blood flow in the corresponding NSR experiment.

The model used in this study has 83 coupled stiff ODEs. An in-house ODE solver [6] was deployed to generate stable and accurate numerical solutions. The maximum integration timestep in the adaptive and implicit solver was $0.001 \mathrm{~s}$, which was found to provide the same solution when timestep was halved. The solutions were obtained using a relative tolerance of $10^{-6}$, with an accuracy of $O\left(\mathrm{dt}^{6}\right)$. Each instance of the simulation could be processed by available computing resources running Red Hat Linux within 2 minutes. 


\section{Results.}

It is observed that large variations in blood pressure propagated through the large arterial circulation and have a high impact on small vessels downstream. This effect is demonstrated in Fig. 3 where a drop in blood pressure due to a long RR interval is associated with two consecutive hypoperfusion events in the left middle (LM) artery.
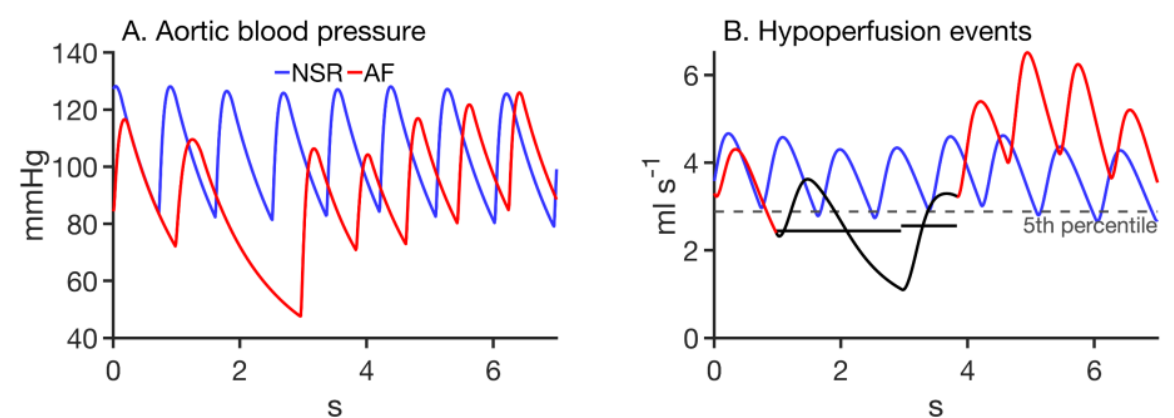

Fig. 3. Hemodynamic outputs of a simulation of AF (red) and NSR (blue) at $70 \mathrm{bpm}$ in the normal CoW. A: Aortic blood pressures. B: Blood flow through the left middle distal artery with hypoperfusion events shown in black.
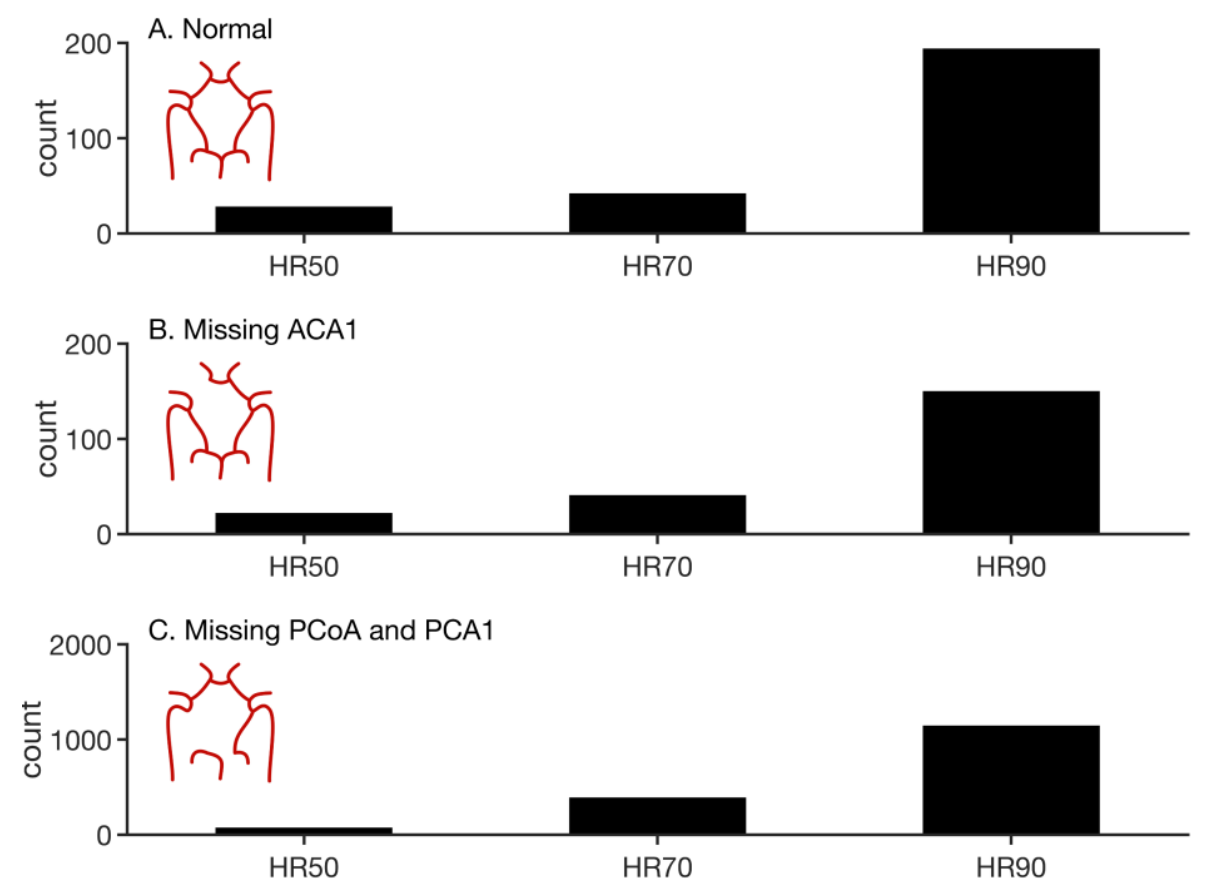

Fig. 4. Absolute frequencies of hypoperfusion events in the distal cerebral circulations at varying heart rates under AF conditions. Histogram stacks are coded according to the 
region they occur in: LM, RM, LA, RA, LP, RP from bottom to top. A: normal CoW. $\mathrm{B}$ : ACA1 variant. C: PCoA and PCA1 variant.

The heart rate and vascular geometry dependence of hypoperfusion events is illustrated in Fig. 4. Three heart rates representing bradycardia (50 bpm), NSR (70 bpm), and tachycardia $(90 \mathrm{bpm})$ were used. Under bradycardia, $33 \%$ less cerebral hypoperfusion events were observed (Fig. 4, A). In contrast, under tachycardia, 362\% more hypoperfusion events were observed (Fig. 4, A). In the missing ACA1 variant, bradycardia reduced hypoperfusion events by $47 \%$, but tachycardia increased the same by $260 \%$ (Fig. 4, B). In case PCoA as well as PCA1 are missing, bradycardia reduced hypoperfusion events by $82 \%$, but tachycardia increased the same by $192 \%$ (Fig. 4, C).

Fig. 5 illustrates cerebral heterogeneity under the three variants. Under NSR conditions (Fig. 5, A), terminals of the left middle, left anterior, and left posterior arteries experiences a balanced outflow, indicating virtually uniform cerebral perfusion. A similar behaviour was observed when the variant with ACA1 artery was missing. However, when PCoA along with PCA1 were missing, the blood flow to terminals was highly heterogeneous.
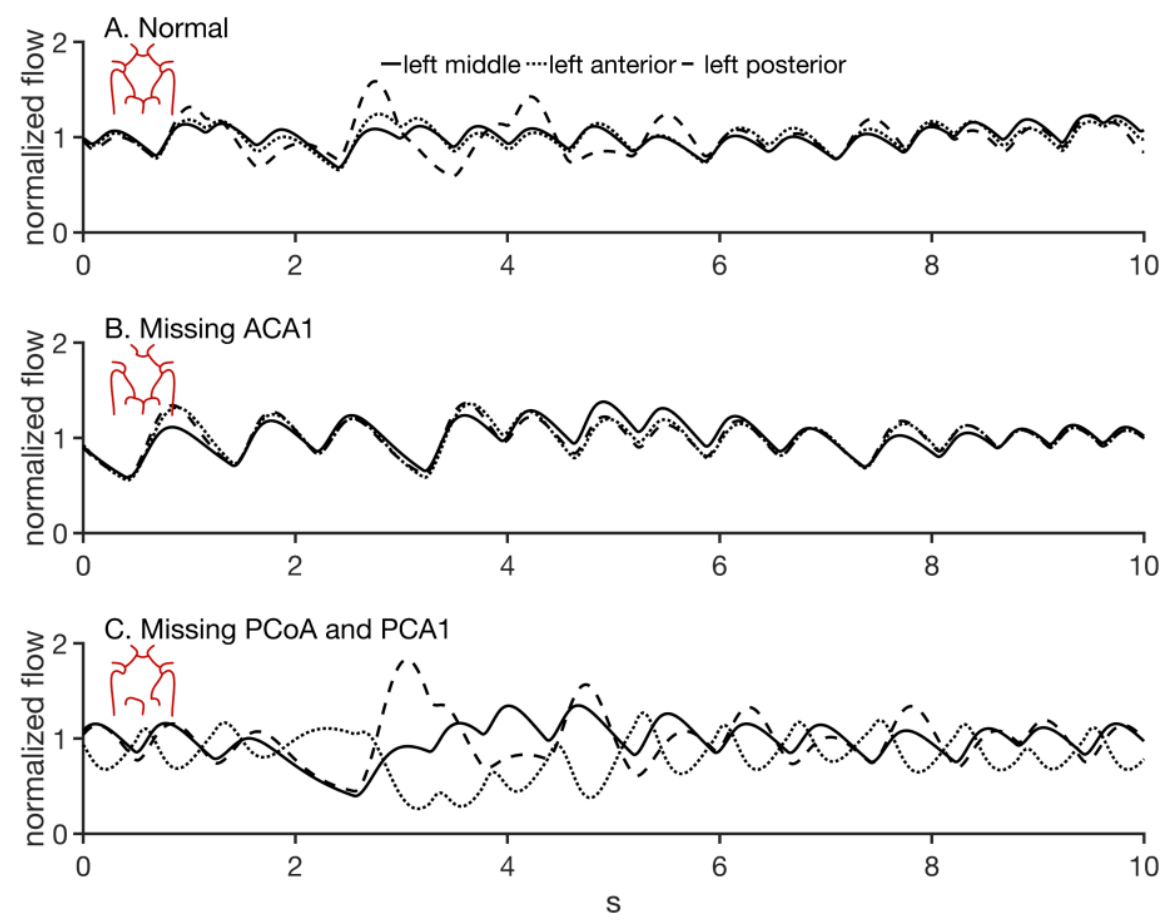

Fig. 5. Perfusion to various parts of the brain, represented by outflow at three distinct vessel terminals. In all panels solid line represents flow at the terminal of left middle artery, dotted lines represents flow at the terminal of left anterior artery, and dashed line represents flow at the terminal of left posterior artery. A: normal CoW. B: ACA1 variant. C: PCoA and PCA1 variant. 


\section{Conclusions and Discussion.}

Variations from regular blood pressure in large arteries due to AF were shown to be associated with large changes in blood flow in the distal circulation of the brain. These changes lead to occurrences of critical hypoperfusion events in the brain, which over time, may lead to damage of the brain tissue. It is clear from the present investigation of different $\mathrm{CoW}$ variants, that individual vascular structure plays a role in the impact of AF on the cerebral circulation. In the case of a missing ACA1 segment, the brain was found to be less prone to hypoperfusion events than normal, while a missing PCoA and contralateral PCA1 led to approximately 5 times the frequency of events. It was also shown that certain structures could lead to increased heterogeneity in cerebral blood flow, with increased blood flow in some regions, and decreased in others. These dependencies on structure demonstrate the need for increased study of cerebral cardiovascular disease with respect to vascular structure. The OD model has been very informative in that it has revealed these phenomena, where current in vivo methods could not. Further investigation will be done using 1D modelling to investigate the impacts of these phenomena on the blood vessels as well as the surrounding tissue in greater detail.

\section{Acknowledgements.}

This work was supported by Canarie Inc. (RS-111), Canada Heart and Stroke Foundation grant (G-20-0028717), Canada NSERC operational grant (R4081A03), and NSERC graduate scholarship. We thank Compute Canada for high performance computing resources. We thank Dr. Kapiraj Chandrabalan for editing support.

\section{References}

[1] Gardarsdottir, M., Sigurdsson, S., Aspelund, T., Rokita, H., Launer, L.J., Gudnason, V., Arnar, D.O.: Atrial fibrillation is associated with decreased total cerebral blood flow and brain perfusion. Europace 20, 1252-1258 (2018)

[2] Anazodo, U.C., Shoemaker, J.K., Suskin, N., St Lawrence, K.S.: An investigation of changes in regional gray matter volume in cardiovascular disease patients, pre and post cardiovascular rehabilitation. NeuroImage. Clinical 3, 388-395 (2013)

[3] Steinman, D.A., Poepping, T.L., Tambasco, M., Rankin, R.N., Holdsworth, D.W.: Flow patterns at the stenosed carotid bifurcation: effect of concentric versus eccentric stenosis. Ann Biomed Eng 28, 415-423 (2000)

[4] Antiga, L., Piccinelli, M., Botti, L., Ene-Iordache, B., Remuzzi, A., Steinman, D.A.: An image-based modeling framework for patient-specific computational hemodynamics. Med Biol Eng Comput 46, 1097-1112 (2008)

[5] Ursino, M., Giannessi, M.: A model of cerebrovascular reactivity including the circle of willis and cortical anastomoses. Ann Biomed Eng 38, 955-974 (2010)

[6] Altamirano-Diaz, L., Kassay, A.D., Serajelahi, B., McIntyre, C.W., Filler, G., Kharche, S.R.: Arterial Hypertension and Unusual Ascending Aortic Dilatation in a Neonate With Acute Kidney 
Injury: Mechanistic Computer Modeling. Frontiers in Physiology 10, (2019)

[7] Anselmino, M., Scarsoglio, S., Saglietto, A., Gaita, F., Ridolfi, L.: Transient cerebral hypoperfusion and hypertensive events during atrial fibrillation: a plausible mechanism for cognitive impairment. Scientific reports 6, 28635 (2016)

[8] Heldt, T., Shim, E.B., Kamm, R.D., Mark, R.G.: Computational modeling of cardiovascular response to orthostatic stress. J Appl Physiol (1985) 92, 1239-1254 (2002)

[9] Heldt, T., Mukkamala, R., Moody, G.B., Mark, R.G.: CVSim: An Open-Source Cardiovascular Simulator for Teaching and Research. The open pacing, electrophysiology \& therapy journal 3, 45-54 (2010)

[10] Lin, J., Ngwompo, R.F., Tilley, D.G.: Development of a cardiopulmonary mathematical model incorporating a baro-chemoreceptor reflex control system. Proceedings of the Institution of Mechanical Engineers. Part H, Journal of engineering in medicine 226, 787-803 (2012)

[11] Alastruey, J., Parker, K.H., Peiro, J., Byrd, S.M., Sherwin, S.J.: Modelling the circle of Willis to assess the effects of anatomical variations and occlusions on cerebral flows. Journal of biomechanics 40, 1794-1805 (2007)

[12] Scarsoglio, S., Guala, A., Camporeale, C., Ridolfi, L.: Impact of atrial fibrillation on the cardiovascular system through a lumped-parameter approach. Med Biol Eng Comput 52, 905 920 (2014)

[13] Hennig, T., Maass, P., Hayano, J., Heinrichs, S.: Exponential distribution of long heart beat intervals during atrial fibrillation and their relevance for white noise behaviour in power spectrum. J Biol Phys 32, 383-392 (2006)

[14] Anselmino, M., Scarsoglio, S., Saglietto, A., Gaita, F., Ridolfi, L.: A Computational Study on the Relation between Resting Heart Rate and Atrial Fibrillation Hemodynamics under Exercise. PLoS ONE 12, e0169967 (2017)

[15] Pianelli, M., Scaglione, M., Anselmino, M., Caponi, D., Garcia, P., Cesarani, F., Toso, E., Raimondo, C., Halimi, F., Leclercq, J.F., Gaita, F.: Delaying cardioversion following 4-week anticoagulation in case of persistent atrial fibrillation after a transcatheter ablation procedure to reduce silent cerebral thromboembolism: a single-center pilot study. J Cardiovasc Med (Hagerstown) 12, 785-789 (2011) 